\title{
Transparencia en la investigación biomédica: A propósito de los riesgos asociados al uso de Avandia
}

\author{
SOFÍA P. SALAS ${ }^{1}$, MOISÉS RUSSO ${ }^{1,2}$
}

\section{Transparency in biomedical research: About the risks associated with the use of Avandia}

The question of how and when to communicate potential risks associated with new drugs has remained an important focus of tension between the pharmaceutical industry and regulatory entities, such as the American Food and Drug Administration. Recently there has been widespread concern related to the cardiovascular risks associated with the use of Avandia, a rosiglitazone produced by Glaxo Smith Kline. In fact, several metaanalyses involving rosiglitazone provided a relatively consistent message that rosiglitazone increases the risk of myocardial ischemic events. In the present article, we review the controversy regarding the way the pharmaceutical company handled this issue, and we describe examples of inappropriate conduct regarding an industry-sponsored clinical trial with this drug. We raise several important ethical questions related to the way researchers handle conflicts of interest when they are employees of the pharmaceutical industry. Finally, we discuss the requirements to conduct biomedical research funded by the pharmaceutical industry in Chile.

(Rev Med Chile 2010; 138: 1197-1200).

Key words: Biomedical research; Conflicts of interest; Rosiglitazone.

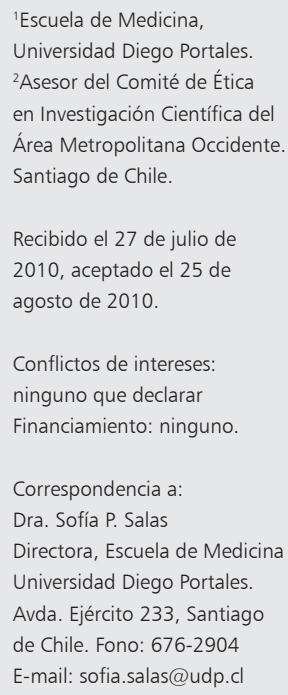

L a pregunta de cómo y cuándo se deben comunicar los posibles riesgos asociados a nuevos fármacos ha mantenido una importante tensión entre la industria farmacéutica y los entes reguladores. Un ejemplo de interés es lo ocurrido con el producto Avandia, de GlaxoSmithKline (GSK), cuyo principio activo, rosiglitazona, usado para controlar la diabetes, parece aumentar el riesgo de muerte cardiovascular ${ }^{1,2}$. En efecto, un reciente estudio de 227.571 pacientes mayores de 65 años tratados con rosiglitazona o pioglitazona, mostró que el producto de GSK tenía mayor riesgo de eventos cardiovasculares adversos, incluyendo insuficiencia cardíaca y muerte, que el fármaco de la competencia ${ }^{3}$.

La rosiglitazona pertenece a la familia de las tiazolidinedionas, y actúa como sensibilizador de la insulina, reduciendo glucosa, ácidos grasos y niveles de insulina en la sangre. En la actualidad, existen al menos dos tiazolidinedionas aprobadas para su uso en los Estados Unidos de Norteamérica: el maleato de rosiglitazona (Avandia) y el hidrocloridrato de pioglitazona (Actos); un tercer tipo (Troglitazona) fue retirado del mercado debido a toxicidad hepática ${ }^{3,4}$. El mecanismo de acción para disminuir los niveles sanguíneos de glucosa se relaciona con su unión a los receptores activados por proliferadores peroxisomales (PPARs), los que se expresan en diversos tejidos. Este mismo mecanismo sería el responsable de algunos de sus efectos adversos, puesto que se activarían los PPARs en el nefrón distal, causando aumento de la reabsorción de sodio y agua ${ }^{3}$. En este sentido, tal como lo señala Juurlink, es conveniente tomar en cuenta que estrategias farmacológicas diseñadas en contra de receptores nucleares tendrán conse- 
cuencias en todos los sitios donde estos receptores se expresen ${ }^{3}$.

Informaciones de prensa señalan que desde hace varios años se tenía conocimiento que los pacientes que usaban Avandia tenían riesgo cardiovascular aumentado ${ }^{5,6}$. En los años 2005 y 2006, meta-análisis realizados por investigadores de GSK encontraron que Avandia aumentaba tres veces el riesgo de problemas cardiovasculares severos. Otros estudios, publicados a fines de 2006, también concordaron con que Avandia aumentaba el riesgo cardiovascular, así como la ganancia de peso y el edema, con respecto a otros hipoglicemiantes con los que fue comparado, pero producía menos eventos gastrointestinales e hipoglicemia ${ }^{7}$. Según el New York Times (NYT), existiría un duro debate al interior de la Food and Drug Administration (FDA) de los EEUU de NA con respecto a la conducta a seguir con Avandia. Mientras algunos aconsejan que se retire del mercado, puesto que existen alternativas más seguras, otros insisten en que los estudios sobre la droga son contradictorios y aún insuficientes para determinar que efectivamente aumenta el riesgo cardiovascular. Aún más preocupante sería la conducta de GSK que habría sostenido prácticas intimidatorias para silenciar a aquellos médicos que señalaban los riesgos del producto $^{5,8,9}$. Recientemente, una investigación oficial de la FDA encontró al menos una docena de casos en los cuales pacientes que tomaban Avandia y que tuvieron serios problemas cardiovasculares, no fueron incluidos en el recuento de eventos adversos de la droga ${ }^{6}$. Por otra parte, el estudio "TIDE", patrocinado por GSK, que intentará contestar la pregunta sobre el riesgo cardiovascular de Avandia, ha sido criticado por el Senador Chuck Grassley, miembro del Comité de Finanzas del Senado Estadounidense, quien señaló que los pacientes en dicho estudio no han sido informados de la sospecha de riesgo cardiovascular aumentado que tiene esta droga ${ }^{10}$. El Senador Grassley hizo una dura crítica a la FDA con respecto a los mecanismos de control de fármacos que están en el mercado, puesto que los mismos encargados de evaluar la seguridad de estos productos están bajo la tutela de los responsables de la aprobación inicial que otorga la FDA, recalcando la necesidad que exista plena independencia para los estudios de efectos adversos que se conocen después de que una droga está en el mercado ${ }^{10}$.

A fines de marzo de este año, la revista JAMA publicó un artículo que comenta el informe del Senado Norteamericano de febrero de este año, el cual incluye copia de intercambio de correos electrónicos y otros documentos internos de GSK ${ }^{8}$. La acusación que hace Nissen en su artículo involucra no sólo a los representantes de GSK sino que también a otros investigadores quienes, actuando como revisores de un artículo de Nissen presentado para publicación en New England Journal of Medicine, violaron flagrantemente cláusulas de confidencialidad al enviar a GSK el artículo en revisión, que indicaba los potenciales riesgos de Avandia. GSK, preocupado por el contenido del artículo de Nissen, lo hizo circular entre sus consejeros científicos, expertos en bioestadística y ejecutivos de alto nivel, para tratar de contrarrestar la evidencia presente en el meta análisis de Nissen, diseñando una estrategia para conservar la participación en el mercado de los hipoglicemiantes. Esta estrategia incluía abrir el ciego de un estudio concurrente (el estudio RECORD), en un intento por publicar antes de tiempo un análisis provisorio no contemplado en el diseño experimental inicial. En este punto, GSK se comunicó con el comité externo de monitoreo de datos (el cual debiera ser independiente y estar en pleno control de los datos del estudio), solicitando autorizar un análisis interino, sin decirles que habían abierto el ciego del estudio dos semanas antes, lo cual prueba que el laboratorio tenía acceso no autorizado a los datos. Sin duda que, tal como lo señala Nissen, la experiencia sobre esta droga y sus fabricantes despiertan preocupaciones con respecto a la relación entre la investigación biomédica y la industria que la financia. En este sentido, aparece como crítico que en el diseño de los ensayos clínicos se contemple la realización de un monitoreo independiente de los datos, que incluya la evaluación por un experto en bioestadística ajeno al patrocinador del estudio ${ }^{8}$.

En una editorial de la misma revista ${ }^{9}$, los autores describieron numerosas situaciones irregulares que rodearon este caso, las que tienen como determinante común la presencia de conflictos de intereses. En primer lugar, está la motivación de uno de los revisores del trabajo, quien violó el compromiso de confidencialidad al enviarlo a GSK; están también los investigadores de dicha industria farmacéutica quienes no respetaron la rigurosidad que amerita un estudio clínico, solicitando un análisis interino no contemplado en el estudio inicial; están los miembros del comité de 
monitoreo de datos, que debieron haber advertido de los riesgos potenciales a los pacientes, negándose a modificar el diseño de un trabajo ya en curso; y también la responsabilidad de los editores de revistas biomédicas, que podrían haber objetado la publicación de estudios con problemas de diseño o sin el poder estadístico suficiente para comprobar la presencia de eventuales eventos adversos serios.

Tal como lo señala De Angelis, el caso de Avandia es un ejemplo de cómo intereses corporativos se sobreponen a los principios científicos y éticos inherentes a la investigación biomédica ${ }^{9}$. Asimismo, la investigación del Senado Norteamericano determinó que GSK debió haber advertido precozmente a los pacientes y los médicos sobre estos efectos adversos ${ }^{10}$. Al parecer, son tan grandes los conflictos de intereses que rodean la investigación biomédica, que es necesario diseñar estrategias para evitar que la información recabada en los protocolos clínicos sea conocida exclusivamente por empleados de la compañía ${ }^{11,12}$. En este sentido, la propuesta del JAMA de exigir que el análisis estadístico sea repetido por un experto independiente de la compañía, preferentemente vinculado a un centro académico, va en el sentido correcto?.

Dada la nueva evidencia obtenida del estudio $\mathrm{RECORD}^{13}$, que confirma el aumento del riesgo cardiovascular en pacientes diabéticos tratados con rosiglitazona y como lo proponen sus autores, este agente debe ser discontinuado en pacientes que desarrollen insuficiencia cardíaca y debe monitorearse muy de cerca su uso en pacientes de edad, en aquellos con aumento del índice de masa corporal, en los que tienen microalbuminuria/proteinuria y también en diabéticos hipertensos. Es importante señalar que algunas sociedades científicas fueron precavidas en su momento y decidieron eliminar Avandia de sus recomendaciones para pacientes diabéticos ${ }^{1}$. Por último, en este caso es necesario reconocer el papel fundamental que han tenido los medios periodísticos al informar los riesgos de este producto a la población de potenciales usuarios y también a los médicos. Recientemente se evaluó el impacto que tuvo el alerta de seguridad que se publicó en la prensa en mayo de 2007 sobre este fármaco, en 552 pacientes de atención primaria en Estados Unidos de Norteamérica que estaban medicados con Avandia ${ }^{14}$. Se determinó que el $62 \%$ discontinuó la terapia como resultado de esta advertencia, ya sea por indicación médica o por decisión del mismo paciente, sin que esto afectara negativamente el control de su diabetes. Por lo tanto, aparece aconsejable estimular a los medios periodísticos para que se mantengan conectados con los entes reguladores y los profesionales de la salud, puesto que proveen de una forma eficaz y oportuna de advertir de los riesgos.

Sería erróneo pensar que el problema descrito se circunscribe a las prácticas de un laboratorio con respecto a un determinado producto. El tema de los conflictos de intereses es más extenso y, tal como señala la reciente propuesta a las sociedades profesionales médicas para controlar la relación entre sus miembros y la industria, afecta casi todo el ámbito de las relaciones entre médicos e industria ${ }^{15}$. Un aspecto relevante que discuten en su propuesta, se refiere a la magnitud de los compromisos económicos que tienen en la actualidad la mayoría de las sociedades médicas científicas, que dependen para su funcionamiento casi íntegramente del aporte que hace la industria de productos médicos, lo que puede afectar su independencia al momento de redactar guías clínicas.

\section{Algunas consideraciones para Chile}

La información descrita debe poner en alerta a los médicos, las autoridades y el público con respecto a la institucionalidad requerida para llevar a cabo investigación biomédica financiada por la industria farmacéutica, en Chile.

Actualmente, en Chile muchos médicos participan como co-investigadores o aportando pacientes para estudios multicéntricos internacionales financiados por la industria farmacéutica. Es común que los Comités de Ética de la Investigación (CEI) nacionales confíen en que los comités de monitoreo de datos incluidos en estudios multicéntricos internacionales son en realidad independientes $y$, por lo tanto, someten la seguridad de los pacientes chilenos a que el análisis de datos por esos comités extra nacionales sea fidedigno. Cabe preguntarse: ¿Deben nuestros CEI locales confiar en las revisiones de datos realizadas en el extranjero? ¿Están los pacientes chilenos expuestos a riesgos más que mínimos al participar en investigación biomédica internacional?

Las preguntas anteriores son de suma importancia y, en este sentido, parece necesario certificar que los CEI nacionales tengan las competencias éticas y científicas necesarias para proteger a nues- 
tra población. Los CEI deben estar compuestos por profesionales con distintos conocimientos y capacidades, además de tener oportunidades de capacitación constante para poder cumplir su función de cautelar los intereses de la población que participa en investigación biomédica ${ }^{16}$. Para que los CEI cuenten con personas con la competencia técnica necesaria y puedan dedicar el tiempo adecuado a la protección de los sujetos de la investigación biomédica, requieren contar con recursos económicos y humanos suficientes, evitando que las labores de fiscalización y protección recaigan solamente en dedicaciones de tiempo voluntarias. Esto incluye, por cierto, la necesidad de poder contar con asesoría remunerada de profesionales con conocimientos avanzados de estadística.

Los CEI requeridos por la Ley $20.120^{17}$ por ahora funcionan por debajo de las necesidades de un país donde sus profesionales de la salud, a medida que nos aproximamos al desarrollo, aportan cada vez más a la creación de conocimiento científico. Es necesario verificar que nuestros salvaguardas para proteger a la población son suficientes, se encuentran adecuadamente constituidos y en pleno funcionamiento.

\section{Referencias}

1. Rosiglitazone no longer recommended. The Lancet 2008; 372: 1520 .

2. Graham DJ, Ouellet-Hellstrom R, Macurdy TE, Ali F, Sholley C, Worrall C, et al. Risk of Acute Myocardial Infarction, Stroke, Heart Failure, and Death in Elderly Medicare Patients Treated With Rosiglitazone or Pioglitazone. JAMA 2010; 304:(doi:10.1001/jama.2010.920).

3. Juurlink DN. Rosiglitazone and the Case for Safety Over Certainty. JAMA 2010; 304:doi:10.1001/jama.2010.954).

4. Norris SL, Carson S. Drug class review: Thiazolidinediones. 2008. Publicado por Oregon Health and Science University. Disponible en http://www.ohsu.edu/drugeffectiveness/reports/final.cfm. [Revisado el 30 mayo 2010].

5. Harris G, Research Ties Diabetes Drug to Heart Woes. Publicado el 19 de febrero 2010 en The New York Times. Disponible en http://www.nytimes.com/2010/02/20/ health/policy/20avandia.html. [Revisado el 30 de mayo 2010].

6. Harris G, Caustic Government Report Deals Blow to Diabetes Drug. Publicado el 9 de julio 2010 en
The New York Times. Disponible en http://www. nytimes.com/2010/07/10/health/10diabetes.html? $\mathrm{r}=2 \& \mathrm{ref}=$ health. [Revisado el 10 de julio 2010]

7. Kahn SE, Haffner SM, Heise MA, Herman WH, Holman RR, Jones NP, et al. Glycemic durability of rosiglitazone, metformin, or glyburide monotherapy. N Engl J Med 2006; 355: 2427-43.

8. Nissen SE. Setting the RECORD Straight. JAMA 2010; 303: 1194-5.

9. DeAngelis CD, Fontanarosa PB. Ensuring integrity in industry-sponsored research: primum non nocere, revisited. JAMA 2010; 303: 1196-8.

10. Grassley C. Grassley Continues Case for Greater Independence in FDA's Post-market Review of Drugs. The United States Senate Committee on Finance. Publicado el 28 abril 2010. Disponible en http://finance.senate.gov/ newsroom $/$ ranking/release/?id=95a2f066-260f-49b38c0a-71elefd790dc. [Revisado el 30 de mayo 2010].

11. Salas SP. [Recommendations of the Association of American Medical Colleges on industry support of medical education]. Rev Med Chile 2008; 136: 1085-6.

12. Romero W, Salas SP. [Disclosure of financial sources and conflicts of interest among research articles published in Revista Medica de Chile]. Rev Med Chile 2007; 135: 473-9.

13. Komajda M, McMurray JJ, Beck-Nielsen H, Gomis R, Hanefeld M, Pocock SJ, et al. Heart failure events with rosiglitazone in type 2 diabetes: data from the RECORD clinical trial. Eur Heart J 2010; 31: 824-31.

14. Orrico KB, Lin JK, Wei A, Yue H. Clinical consequences of disseminating the rosiglitazone FDA safety warning. Am J Manag Care 2010; 16: e111-6.

15. Rothman DJ, McDonald WJ, Berkowitz CD, Chimonas SC, DeAngelis CD, Hale RW, et al. Professional medical associations and their relationships with industry: a proposal for controlling conflict of interest. JAMA 2009; 301: 1367-72.

16. Organización de las Naciones Unidas para la Educación, la Ciencia, y la Cultura. Creación de Comités de Bioética, Guía No 1 2005. Disponible en http://www.unesco.org. uy/shs/es/areas-de-trabajo/ciencias-sociales/bioetica/ documentos-publicaciones-en-bioetica.html. [Revisado el 7 de junio 2010].

17. Ministerio de Salud de Chile, Subsecretaría de Salud Pública. Ley 20.120 Sobre la investigación científica en el ser humano, su genoma, y prohíbe la clonación humana. 2006. Disponible en http://www.leychile.cl/ Navegar?idNorma $=253478 \& \mathrm{r}=1$. [Revisado el 7 de junio 2010]. 